$$
\begin{array}{ll}
\text { Research Square } & \begin{array}{l}
\text { Preprints are preliminary reports that have not undergone peer review. } \\
\text { They should not be considered conclusive, used to inform clinical practice, } \\
\text { or referenced by the media as validated information. }
\end{array}
\end{array}
$$

\title{
Use of geographical information systems in multiple sclerosis research: A systematic scoping review
}

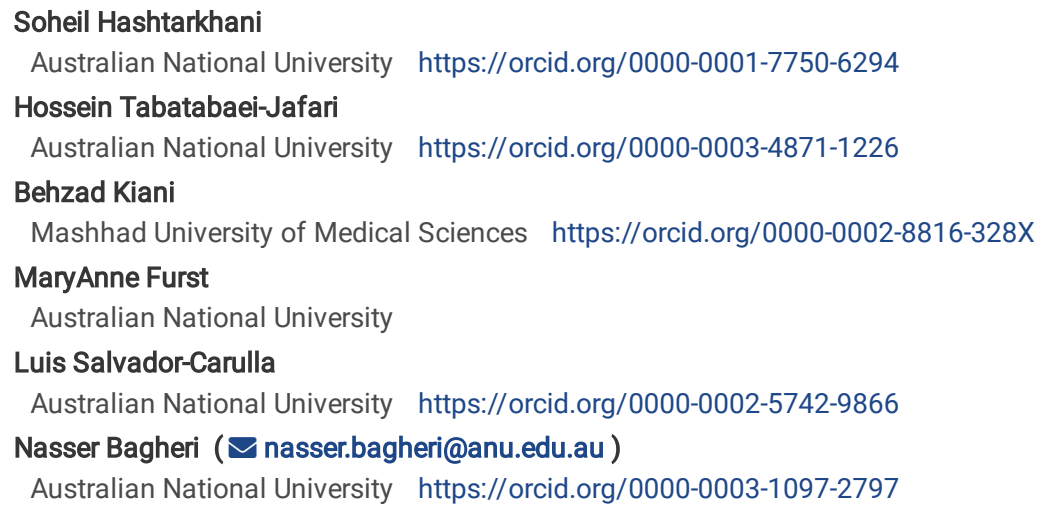

\section{Systematic Review}

Keywords: Multiple Sclerosis, geographical information systems, spatial analysis, health geography

Posted Date: February 12th, 2021

DOI: https://doi.org/10.21203/rs.3.rs-236908/v1

License: (c) (1) This work is licensed under a Creative Commons Attribution 4.0 International License. Read Full License 


\begin{abstract}
Introduction: Geographical Information System (GIS) and spatial analysis have an emerging role in the understanding and management of health-related outcomes. However, there is a knowledge gap about the extent to which GIS has supported Multiple Sclerosis (MS) research. Therefore, this review aimed to explore the types of GIS applications and the complexity of their visualisation in MS research.

Methods: A systematic scoping review was conducted based on York's five-stage framework. PubMed, Scopus and Web of Science were searched for relevant studies published between 2000 and 2020 using a comprehensive search strategy based on the main concepts related to GIS and MS. Grounded, inductive analysis was conducted to organize studies into meaningful application areas. Further, we developed a tool to assess the visualisation complexity of the selected papers.
\end{abstract}

Results: Of 3,723 identified unique citations, 42 papers met our inclusion criteria for the final review. One or more of the following types of GIS applications were reported by these studies: (a) thematic mapping (37 papers); (b) spatial cluster detection (16 papers); (c) risk factors detection (16 papers); and (d) health access and planning (two papers). In the majority of studies (88\%), the score of visualisation complexity was relatively low: three or less from the range of zero to six.

Conclusions: Although the number of studies using GIS techniques has dramatically increased in the last decade, the use of GIS in the areas of MS access and planning is still under-researched. Additionally, the capacity of GIS in visualising complex nature of MS care system is not yet fully investigated.

\title{
1. Introduction
}

Multiple sclerosis (MS) is an autoimmune disease and the most common disabling neurological condition of young adults globally (1). Most people are diagnosed between the ages of 20 and 40, during their most productive years (2). Global prevalence of MS was estimated to be 2-2.5 million cases in 2016, an increase of $10.4 \%$ since 1990 (3). In addition to genetic profile, environmental and lifestyle factors are also important in the development of MS (4-6).

Geographical Information Systems (GIS) are a type of information system designed to collect, store, manage, analyse and display spatial data (7). In the health context, GIS enables researchers to better understand disease patterns over space and time, as well as risk factors and geographic outcome disparities (8). GIS and geospatial modelling can also help policy makers to identify areas of unmet need and to design tailored interventions (9-11).

To date, there is no synthesis of evidence on the utilization of GIS in MS research, which is a significant knowledge gap. To bridge this gap, we conducted a scoping review with a systematic search strategy to synthesise information on the applications of GIS in MS research. A scoping review provides the means to comprehensively synthesise the literature. It is aimed at mapping the evidence and identifying knowledge gaps within the primary studies (12). The aims of this study were: (a) to provide a comprehensive analysis of current literature with applications of GIS in MS research and (b) to describe the GIS techniques used in each type of GIS applications in MS.

\section{Material And Methods}

This review was conducted according to the York five-stage framework proposed by Arksey and O’Malley (12).

\subsection{Identifying the research question}

We defined the main research question as: to what extent, and how, does GIS technology support multiple sclerosis research?

\subsection{Identifying studies}

A preliminary search of the literature was conducted to identify search terms and refine the selection criteria. A comprehensive search strategy was developed using the combination of key words and MeSH terms, ("Geographical Information System" OR spatial OR GIS OR geograph*) AND ("Multiple Sclerosis" OR "MS patients" OR “MS disease"), to retrieve all MS studies applying GIS approaches in the PubMed, Web of Science and Scopus.

All the retrieved citations published in peer-reviewed journals between 1/1/2000 and 23/3/2020 were imported into Endnote X9 software in order to manage and share between reviewers effectively. To boost our search, bibliographies of included studies were manually searched to identify additional studies for inclusion.

\subsection{Selecting studies}

To be selected, articles should be original studies published in peer-reviewed journals in the English language, and use at least one GIS or spatial analysis technique. Two reviewers (M.F. and H.T-J.) screened papers by titles and abstracts and excluded all irrelevant papers. Another two reviewers (SH and BK) scanned the full-texts of all remaining articles against the selection criteria. Another researcher (NB) double-screened the included/excluded full texts to ensure that all relevant studies were included in the review.

\subsection{Charting the data}


A data extraction form was developed using Microsoft Excel 2016, consisting of 20 variables including Yes/No fields, combo boxes and free text fields. Two reviewers (B.K. and S.H.) extracted data from all included articles and any disagreement was resolved by consensus (including N.B. and H.T-J.). Studies were assessed for the type of GIS application used and the complexity of information presented in visualisation maps.

Type of GIS application: The selected studies were scanned for the use of one or more of the following types of GIS application: a) thematic mappingvisualising geographical distribution of MS or related attributes in a geographical map; b) spatial cluster detection-identifying areas of high-risk (or low-risk) of MS; c) risk factors detection- identifying environmental and geographical risk factors associated with MS; and d) health access and planning- measuring accessibility to health services or using new technologies to track and monitor patients' physical activities.

Complexity of GIS visualisations: GIS visualisations in included studies were assessed for the level of complexity and for the information provided. Studies could be potentially scored from zero to six based on the presence of the following items: a) thematic map; b) having overlaid two or more information layers; c) spatio-temporal analysis- showing the temporal pattern of events in addition to spatial distribution in areas; d) spatial clustering-showing statistically significant clusters of events in the map; e) smoothing/heat map analysis-using smoothing techniques or heat map to reduce or cancel the effect of random variation; and f) buffer visualisation- showing distance or travel time buffer in the map. We assigned zero if any of the selected studies had no GIS visualization and one for each aforementioned item. A paper could receive a maximum score of six if it meets all items.

\subsection{Collating, summarising and reporting results}

Microsoft Excel 2016 and ArcGIS 10.6 were used for creating the charts and figures of the report. The distribution of included studies was located on a world choropleth map of MS prevalence. MS prevalence data was extracted from the Global Burden of Disease study (GBD) of MS published in 2019 (3). A Venn diagram was used to visualize the frequency and overlaps of the types of GIS application used across the included studies.

\section{Results}

\section{- 3.1 Included studies and their characteristics}

The literature search identified 3,723 unique citations. After exclusion of irrelevant studies based on title and abstracts, 98 publications remained for full-text assessment. Of these, a further 55 papers did not meet the selection criteria and were excluded. Therefore, 43 articles were reviewed in this scoping review (figure 1). No study was found in the manual-search of the bibliographies of included studies.

Of the 43 included studies, 38 used data from a single country and five studies used data from two or more countries. Single-country studies originated from 13 different countries across three continents: 20 of these were within Europe, 10 in North America, six in Asia, and two were from the Oceania region (Figure 2). MS prevalence in these countries was greater than 68 people per 100,000 populations. Most of the European studies were from western and northern Europe, and five of the six studies in Asia were from Iran. North American studies were conducted in the USA and Canada. Both studies in the Oceania region were conducted in Australia.

As expected, the number of studies has increased over time, particularly since 2008 (Figure 3a). Figure $3 \mathrm{~b}$ reveals that the geographical scale differed across included studies from a city to a whole country. Of the 43 studies, three (7\%) collected data from city level, 19 (44\%) from a state/provincial level, and 16 (37\%) from whole country level. Five studies (12\%) collected data from more than one country.

\section{- 3.2 Type of GIS application}

Since each study could employ more than one GIS application, the list of papers in each group was not mutually exclusive (Figure 4). Figure 4a highlights the number of studies in each GIS application group and the overlap between the groups. The majority of studies $(n=30,69 \%)$ employed more than one type of GIS applications: 29 studies (67\%) employed two types, and one study (2\%) employed three types. Thirteen studies (30\%) employed only one type of GIS applications. Figure $4 \mathrm{~b}$ shows the total number of papers in each group. Thematic mapping was the most commonly used GIS application, employed by 40 included studies (93\%), followed by spatial cluster detection and risk factors detection, each used in 16 studies (37\%), while health access and planning was applied in only two studies (4\%).

Table 1 provides a summary and detailed information on the included studies and distribution of the studies across the defined groups.

Table 1: Characteristics of included studies. 


\begin{tabular}{|c|c|c|c|c|c|c|c|c|c|c|}
\hline \multirow[t]{2}{*}{ ID } & \multirow[t]{2}{*}{ Author, (Year) } & \multicolumn{4}{|c|}{ Type of GIS application } & \multirow{2}{*}{$\begin{array}{l}\text { Visualisation } \\
\text { complexity } \\
\text { score }\end{array}$} & \multirow[t]{2}{*}{ Location } & \multirow[t]{2}{*}{ Size, Time } & \multirow{2}{*}{$\begin{array}{l}\text { Main spatial } \\
\text { analysis }\end{array}$} & \multirow[t]{2}{*}{1} \\
\hline & & $\begin{array}{l}\text { Thematic } \\
\text { mapping }\end{array}$ & $\begin{array}{l}\text { Spatial } \\
\text { cluster } \\
\text { detection }\end{array}$ & $\begin{array}{l}\text { Risk factors } \\
\text { detection }\end{array}$ & $\begin{array}{l}\text { Health } \\
\text { access } \\
\text { and } \\
\text { planning }\end{array}$ & & & & & \\
\hline 1 & $\begin{array}{l}\text { Landtblom, } \\
(2002)(13)\end{array}$ & $\begin{array}{l}\text { Choropleth } \\
\text { map }\end{array}$ & $x$ & $x$ & $x$ & 1 & Sweden & $\begin{array}{l}5245,1952- \\
1992\end{array}$ & $\begin{array}{l}\text { Visualising } \\
\text { mortality rate } \\
\text { and disability } \\
\text { pensioning rate } \\
\text { of MS over the } \\
\text { time }\end{array}$ & $\begin{array}{l}1 \\
c \\
r \\
r\end{array}$ \\
\hline 2 & $\begin{array}{l}\text { Llorca (2005) } \\
(14)\end{array}$ & $\begin{array}{l}\text { Choropleth } \\
\text { map }\end{array}$ & $\mathrm{x}$ & $x$ & $x$ & 2 & Spain & $\begin{array}{l}3084,1975- \\
1998\end{array}$ & $\begin{array}{l}\text { Visualising age- } \\
\text { adjusted MS } \\
\text { mortality }\end{array}$ & $\begin{array}{l}t \\
c \\
c\end{array}$ \\
\hline 3 & $\begin{array}{l}\text { Yiannakoulias } \\
(2007)(15)\end{array}$ & $\begin{array}{l}\text { Choropleth } \\
\text { map }\end{array}$ & $x$ & $x$ & $x$ & 1 & $\begin{array}{l}\text { Alberta, } \\
\text { Canada }\end{array}$ & $\begin{array}{l}7602,1994- \\
2004\end{array}$ & $\begin{array}{l}\text { Visualising } \\
\text { percentage } \\
\text { change in } \\
\text { distribution of } \\
\text { MS cases } \\
\text { between index } \\
\text { date and } 2004\end{array}$ & $\begin{array}{l}\mathrm{F} \\
\hat{j} \\
\mathrm{i} \\
\mathrm{c} \\
\mathrm{r} \\
\mathrm{r} \\
\mathrm{F}\end{array}$ \\
\hline 4 & $\begin{array}{l}\text { Bostrom } \\
(2009)(16)\end{array}$ & $\begin{array}{l}\text { Choropleth } \\
\text { map }\end{array}$ & $x$ & $\mathrm{x}$ & $x$ & 1 & $\begin{array}{l}\text { Varmland, } \\
\text { Sweden }\end{array}$ & $\begin{array}{l}465,2002- \\
2003\end{array}$ & $\begin{array}{l}\text { Visualising } \\
\text { prevalence rate } \\
\text { of MS }\end{array}$ & $\begin{array}{l}s \\
F \\
r\end{array}$ \\
\hline 5 & $\begin{array}{l}\text { Palmer (2013) } \\
\text { (17) }\end{array}$ & $\begin{array}{l}\text { Choropleth } \\
\text { map }\end{array}$ & $x$ & $x$ & $x$ & 1 & Australia & $\begin{array}{l}21283 \\
2010\end{array}$ & $\begin{array}{l}\text { Visualising age- } \\
\text { adjusted } \\
\text { prevalence rate } \\
\text { of MS }\end{array}$ & $\begin{array}{l}l \\
F \\
c \\
\epsilon\end{array}$ \\
\hline 6 & $\begin{array}{l}\text { Ramagopalan } \\
(2011)(18)\end{array}$ & $\begin{array}{l}\text { Choropleth } \\
\text { map }\end{array}$ & $\mathrm{x}$ & $x$ & $x$ & 1 & England & $\begin{array}{l}\text { 56681, } \\
1999-2005\end{array}$ & $\begin{array}{l}\text { Visualising } \\
\text { admission Rate } \\
\text { of MS }\end{array}$ & $\begin{array}{l}c \\
\epsilon \\
l\end{array}$ \\
\hline 7 & $\begin{array}{l}\text { Risberg } \\
(2011)(19)\end{array}$ & $\begin{array}{l}\text { Choropleth } \\
\text { map }\end{array}$ & $x$ & $x$ & $\mathrm{x}$ & 1 & $\begin{array}{l}\text { Oppland, } \\
\text { Norway }\end{array}$ & $\begin{array}{l}474,1989- \\
2001\end{array}$ & $\begin{array}{l}\text { Visualising } \\
\text { prevalence and } \\
\text { Incidence of } \\
\text { MS }\end{array}$ & $\begin{array}{l}1 \\
\mathrm{~F} \\
\mathrm{r} \\
\epsilon\end{array}$ \\
\hline 8 & $\begin{array}{l}\text { Bargagli } \\
(2016)(20)\end{array}$ & $\begin{array}{l}\text { Choropleth } \\
\text { map }\end{array}$ & $x$ & $x$ & $x$ & 1 & Lazio, Italy & $\begin{array}{l}7377,2006- \\
2011\end{array}$ & $\begin{array}{l}\text { Visualising age- } \\
\text { and gender- } \\
\text { adjusted MS } \\
\text { prevalence rate }\end{array}$ & $\begin{array}{l}1 \\
r \\
r \\
f\end{array}$ \\
\hline 9 & $\begin{array}{l}\text { McDonald } \\
(2019)(21)\end{array}$ & $\begin{array}{l}\text { Choropleth } \\
\text { map }\end{array}$ & $x$ & $x$ & $x$ & 1 & Scotland & 2569 & $\begin{array}{l}\text { Visualising } \\
\text { incidence of } \\
\text { MS }\end{array}$ & $\begin{array}{l}1 \\
1 \\
1\end{array}$ \\
\hline 10 & $\begin{array}{l}\text { Wallin (2019) } \\
\text { (3) }\end{array}$ & $\begin{array}{l}\text { Choropleth } \\
\text { map }\end{array}$ & $x$ & $x$ & $x$ & 1 & International & $\begin{array}{l}\text { I2221188, } \\
1990-2016\end{array}$ & $\begin{array}{l}\text { Visualising age- } \\
\text { standardised } \\
\text { multiple } \\
\text { sclerosis } \\
\text { prevalence }\end{array}$ & $\begin{array}{l}F \\
i \\
s \\
r \\
s\end{array}$ \\
\hline 11 & $\begin{array}{l}\text { Pugliatti } \\
(2002)(22)\end{array}$ & $\begin{array}{l}\text { Choropleth } \\
\text { map }\end{array}$ & $\begin{array}{l}\text { Bayesian } \\
\text { hierarchical } \\
\text { modelling }\end{array}$ & $x$ & $x$ & 3 & $\begin{array}{l}\text { Northern } \\
\text { Sardinia, Italy }\end{array}$ & 686,1997 & $\begin{array}{l}\text { Discover } \\
\text { spatial } \\
\text { hotspots using } \\
\text { Bayesian } \\
\text { mapping }\end{array}$ & $\begin{array}{l}F \\
\epsilon \\
\epsilon \\
F \\
F\end{array}$ \\
\hline
\end{tabular}




\begin{tabular}{|c|c|c|c|c|c|c|c|c|c|}
\hline 12 & $\begin{array}{l}\text { Bergamaschi } \\
(2006)(23)\end{array}$ & $\begin{array}{l}\text { Choropleth } \\
\text { map }\end{array}$ & $\begin{array}{l}\text { Bayesian } \\
\text { hierarchical } \\
\text { modelling }\end{array}$ & $x$ & $x$ & 3 & Pavia, Italy & 464,2000 & $\begin{array}{l}\text { Discover } \\
\text { spatial } \\
\text { hotspots using } \\
\text { Bayesian } \\
\text { modelling }\end{array}$ \\
\hline 13 & $\begin{array}{l}\text { Cocco (2011) } \\
\text { (24) }\end{array}$ & $\begin{array}{l}\text { Choropleth } \\
\text { map }\end{array}$ & $\begin{array}{l}\text { Bayesian } \\
\text { hierarchical } \\
\text { modelling }\end{array}$ & $x$ & $x$ & 3 & $\begin{array}{l}\text { South-western } \\
\text { Sardinia, Italy }\end{array}$ & $\begin{array}{l}292 \\
12 / 31 / 2007\end{array}$ & $\begin{array}{l}\text { Discover } \\
\text { spatial } \\
\text { hotspots using } \\
\text { Bayesian } \\
\text { modelling }\end{array}$ \\
\hline 14 & $\begin{array}{l}\text { Fromont } \\
(2012)(25)\end{array}$ & $\begin{array}{l}\text { Choropleth } \\
\text { map }\end{array}$ & $\begin{array}{l}\text { Bayesian } \\
\text { hierarchical } \\
\text { modelling }\end{array}$ & $x$ & $x$ & 3 & France & $\begin{array}{l}28682 \\
2000-2007\end{array}$ & $\begin{array}{l}\text { Discover } \\
\text { spatial } \\
\text { hotspots using } \\
\text { Bayesian } \\
\text { modelling }\end{array}$ \\
\hline 15 & $\begin{array}{l}\text { Pivot (2016) } \\
\text { (26) }\end{array}$ & $\begin{array}{l}\text { Choropleth } \\
\text { map }\end{array}$ & $\begin{array}{l}\text { Bayesian } \\
\text { hierarchical } \\
\text { modelling }\end{array}$ & $x$ & $x$ & 3 & France & $\begin{array}{l}\text { Not } \\
\text { mentioned, } \\
12 / 31 / 2004\end{array}$ & $\begin{array}{l}\text { Discover } \\
\text { spatial } \\
\text { hotspots using } \\
\text { Bayesian } \\
\text { modelling }\end{array}$ \\
\hline 16 & $\begin{array}{l}\text { Bezzini } \\
(2017)(27)\end{array}$ & $\begin{array}{l}\text { Choropleth } \\
\text { map }\end{array}$ & $\begin{array}{l}\text { Bayesian } \\
\text { hierarchical } \\
\text { modelling }\end{array}$ & $x$ & $x$ & 3 & Tuscany, Italy & $\begin{array}{l}7330 \\
12 / 31 / 2013\end{array}$ & $\begin{array}{l}\text { Discover } \\
\text { spatial } \\
\text { hotspots using } \\
\text { Bayesian } \\
\text { modelling }\end{array}$ \\
\hline 17 & $\begin{array}{l}\text { Bergamaschi } \\
(2019)(28)\end{array}$ & $\begin{array}{l}\text { Choropleth } \\
\text { map }\end{array}$ & $\begin{array}{l}\text { Bayesian } \\
\text { hierarchical } \\
\text { modelling }\end{array}$ & $x$ & $x$ & 3 & Northern Italy & $\begin{array}{l}927 \\
12 / 31 / 2016\end{array}$ & $\begin{array}{l}\text { Discover } \\
\text { spatial } \\
\text { hotspots using } \\
\text { Bayesian } \\
\text { modelling }\end{array}$ \\
\hline 18 & $\begin{array}{l}\text { Fromont } \\
(2010)(29)\end{array}$ & $\begin{array}{l}\text { Choropleth } \\
\text { map }\end{array}$ & $\begin{array}{l}\text { Bayesian } \\
\text { hierarchical } \\
\text { modelling }\end{array}$ & $x$ & $x$ & 3 & France & $\begin{array}{l}\text { 49417, } \\
2003-2004\end{array}$ & $\begin{array}{l}\text { Discover } \\
\text { spatial } \\
\text { hotspots using } \\
\text { Bayesian } \\
\text { modelling }\end{array}$ \\
\hline
\end{tabular}




\begin{tabular}{|c|c|c|c|c|c|c|c|c|c|}
\hline 19 & $\begin{array}{l}\text { Green (2013) } \\
(30)\end{array}$ & $\begin{array}{l}\text { Choropleth } \\
\text { map }\end{array}$ & $\begin{array}{l}\text { Bayesian } \\
\text { hierarchical } \\
\text { modelling, } \\
\text { spatial } \\
\text { scan } \\
\text { statistics }\end{array}$ & $x$ & $x$ & 3 & Canada & $\begin{array}{l}2290,1990- \\
2006\end{array}$ & $\begin{array}{l}\text { Discover } \\
\text { spatial } \\
\text { hotspots using } \\
\text { Bayesian } \\
\text { modelling and } \\
\text { spatial scan } \\
\text { statistics }\end{array}$ \\
\hline 20 & $\begin{array}{l}\text { Torabi (2014) } \\
\text { (31) }\end{array}$ & $\begin{array}{l}\text { Choropleth } \\
\text { map }\end{array}$ & $\begin{array}{l}\text { Bayesian } \\
\text { hierarchical } \\
\text { modelling, } \\
\text { spatial } \\
\text { scan } \\
\text { statistics }\end{array}$ & $x$ & $\mathrm{x}$ & 3 & $\begin{array}{l}\text { Manitoba, } \\
\text { Canada }\end{array}$ & $\begin{array}{l}2290,1990- \\
2006\end{array}$ & $\begin{array}{l}\text { Discover } \\
\text { spatial } \\
\text { hotspots using } \\
\text { Bayesian } \\
\text { mapping and } \\
\text { spatial scan } \\
\text { statistics }\end{array}$ \\
\hline 21 & $\begin{array}{l}\text { Donnan } \\
(2005)(32)\end{array}$ & $\begin{array}{l}\text { Choropleth } \\
\text { map }\end{array}$ & $\begin{array}{l}\text { Spatial } \\
\text { scan } \\
\text { statistics }\end{array}$ & $x$ & $x$ & 2 & $\begin{array}{l}\text { Tayside, } \\
\text { Scotland }\end{array}$ & $\begin{array}{l}772,1970- \\
1997\end{array}$ & $\begin{array}{l}\text { Discover } \\
\text { spatial and } \\
\text { temporal } \\
\text { hotspots using } \\
\text { spatial scan } \\
\text { statistics }\end{array}$ \\
\hline 22 & $\begin{array}{l}\text { Turabelidze } \\
(2008)(33)\end{array}$ & $\begin{array}{l}\text { Choropleth } \\
\text { map }\end{array}$ & $\begin{array}{l}\text { Spatial } \\
\text { scan } \\
\text { statistics }\end{array}$ & $x$ & $x$ & 2 & USA & $\begin{array}{l}321,1998- \\
2002\end{array}$ & $\begin{array}{l}\text { Discover } \\
\text { spatial } \\
\text { hotspots using } \\
\text { spatial scan } \\
\text { statistics }\end{array}$ \\
\hline 23 & $\begin{array}{l}\text { Saei (2014) } \\
\text { (34) }\end{array}$ & $\begin{array}{l}\text { Choropleth } \\
\text { map, Heat } \\
\text { map }\end{array}$ & $\begin{array}{l}\text { Getis-Ord } \\
\mathrm{Gi}^{*}\end{array}$ & $x$ & $x$ & 3 & Tehran, Iran & $\begin{array}{l}6027,2001- \\
2012\end{array}$ & $\begin{array}{l}\text { Discover } \\
\text { spatial } \\
\text { hotspots using } \\
\text { Getis-Ord Gi* } \\
\text { test }\end{array}$ \\
\hline 24 & $\begin{array}{l}\text { Bihrmann } \\
(2018)(35)\end{array}$ & Heat map & $\begin{array}{l}\text { Kernel } \\
\text { regression }\end{array}$ & $x$ & $x$ & 4 & Denmark & $\begin{array}{l}\text { 12993, } \\
1971-2013\end{array}$ & $\begin{array}{l}\text { Discover } \\
\text { spatial } \\
\text { hotspots using } \\
\text { kernel } \\
\text { regression }\end{array}$ \\
\hline 25 & $\begin{array}{l}\text { Wade (2014) } \\
\text { (36) }\end{array}$ & $\begin{array}{l}\text { Choropleth } \\
\text { map }\end{array}$ & $\begin{array}{l}\text { Local and } \\
\text { global } \\
\text { Moran's, } \\
\text { Getis-Ord } \\
\text { General G }\end{array}$ & $x$ & $x$ & 2 & International & $\begin{array}{l}131 \\
\text { prevalence } \\
\text { study }\end{array}$ & $\begin{array}{l}\text { Using local and } \\
\text { global hotspot } \\
\text { analysis of } \\
\text { Local Moran's } \\
\text { and Getis-Ord } \\
\text { General G }\end{array}$ \\
\hline 26 & $\begin{array}{l}\text { Gregory } \\
(2008)(37)\end{array}$ & $\begin{array}{l}\text { Choropleth } \\
\text { map }\end{array}$ & $x$ & $\begin{array}{l}\text { Per capita } \\
\text { income, and } \\
\text { PM-10 }\end{array}$ & $x$ & 1 & Georgia, USA & 6247,2006 & $\begin{array}{l}\text { Bivariate linear } \\
\text { regression to } \\
\text { find } \\
\text { association }\end{array}$ \\
\hline
\end{tabular}




\begin{tabular}{|c|c|c|c|c|c|c|c|c|c|}
\hline 27 & $\begin{array}{l}\text { Sloka (2008) } \\
\text { (38) }\end{array}$ & $\begin{array}{l}\text { Choropleth } \\
\text { map }\end{array}$ & $x$ & $\begin{array}{l}\text { Ultraviolet B } \\
\text { (UVB) }\end{array}$ & $x$ & 1 & $\begin{array}{l}\text { Newfoundland, } \\
\text { Canada }\end{array}$ & $\begin{array}{l}328,1996- \\
2003\end{array}$ & $\begin{array}{l}\text { Bayesian } \\
\text { mapping to find } \\
\text { geographical } \\
\text { distribution and } \\
\text { covariate } \\
\text { analysis to find } \\
\text { associations } \\
\text { and } \\
\text { interpolation to } \\
\text { estimate } \\
\text { unknown } \\
\text { values of UVB }\end{array}$ \\
\hline 28 & $\begin{array}{l}\text { Beretich } \\
(2009)(39)\end{array}$ & $\begin{array}{l}\text { Proportional } \\
\text { map }\end{array}$ & $x$ & UV index & $x$ & 2 & North America & $\begin{array}{l}\text { Data } \\
\text { obtained } \\
\text { from other } \\
\text { studies }\end{array}$ & $\begin{array}{l}\text { bivariate linear } \\
\text { regression }\end{array}$ \\
\hline 29 & $\begin{array}{l}\text { Amram } \\
(2018)(40)\end{array}$ & $x$ & $x$ & UVB & $x$ & 0 & Canada & $\begin{array}{l}3226,1980- \\
2005\end{array}$ & $\begin{array}{l}\text { Remote } \\
\text { sensing and } \\
\text { basic GIS } \\
\text { analysis } \\
\text { (Overlay) }\end{array}$ \\
\hline 30 & $\begin{array}{l}\text { Handel (2010) } \\
(41)\end{array}$ & Heat map & $x$ & $\begin{array}{l}\text { UV index, } \\
\text { smoking }\end{array}$ & $x$ & 3 & Europe & $\begin{array}{l}\text { Data } \\
\text { obtained } \\
\text { from other } \\
\text { studies }\end{array}$ & $\begin{array}{l}\text { Linear } \\
\text { regression to } \\
\text { find } \\
\text { associations }\end{array}$ \\
\hline 31 & $\begin{array}{l}\text { Taylor (2010) } \\
(42)\end{array}$ & $x$ & $x$ & Latitude & $x$ & 0 & Australia & $\begin{array}{l}330,2003- \\
2006\end{array}$ & $\begin{array}{l}\text { Poisson } \\
\text { regression to } \\
\text { find } \\
\text { associations }\end{array}$ \\
\hline 32 & $\begin{array}{l}\text { Ramagopalan } \\
(2011)(43)\end{array}$ & $\begin{array}{l}\text { Choropleth } \\
\text { map }\end{array}$ & $x$ & $\begin{array}{l}\text { UVB and } \\
\text { infectious } \\
\text { mononucleosis }\end{array}$ & $x$ & 1 & England & $\begin{array}{l}\text { Not } \\
\text { mentioned, } \\
\text { 1998-2005 }\end{array}$ & $\begin{array}{l}\text { Geographical } \\
\text { Weighted } \\
\text { Regression to } \\
\text { find } \\
\text { associations }\end{array}$ \\
\hline 33 & $\begin{array}{l}\text { Tsai (2013) } \\
\text { (44) }\end{array}$ & $\begin{array}{l}\text { Choropleth } \\
\text { map }\end{array}$ & $x$ & $\begin{array}{l}\text { Arsenic (As), } \\
\text { Mercury (Hg), } \\
\text { Cadmium (Cd), } \\
\text { Chromium (Cr), } \\
\text { Copper (Cu), } \\
\text { Nickel (Ni), } \\
\text { Lead (Pb) and } \\
\text { Zinc ( } \mathrm{Zn})\end{array}$ & $x$ & 2 & Taiwan & $\begin{array}{l}1240,1997- \\
2008\end{array}$ & $\begin{array}{l}\text { Interpolation } \\
\text { (IDW method) } \\
\text { and spatial } \\
\text { regression to } \\
\text { find } \\
\text { associations }\end{array}$ \\
\hline 34 & $\begin{array}{l}\text { Schuurman } \\
(2013)(45)\end{array}$ & $\begin{array}{l}\text { Bar graph } \\
\text { map }\end{array}$ & $x$ & $\begin{array}{l}\text { Ultraviolet B } \\
\text { (UVB) }\end{array}$ & $x$ & 1 & Canada & 4010,2005 & $\begin{array}{l}\text { Raster } \\
\text { Resampling } \\
\text { and Overlay }\end{array}$ \\
\hline
\end{tabular}




\begin{tabular}{|c|c|c|c|c|c|c|c|c|c|c|}
\hline 35 & $\begin{array}{l}\text { Heydarpour } \\
(2014)(46)\end{array}$ & $\begin{array}{l}\text { Heat map, } \\
\text { Dot density } \\
\text { map }\end{array}$ & $\begin{array}{l}\text { average } \\
\text { nearest } \\
\text { neighbour } \\
\text { index }\end{array}$ & $\begin{array}{l}\mathrm{PM}-10, \mathrm{SO} 2, \\
\mathrm{NO}, \mathrm{NO} 2, \mathrm{NOX}\end{array}$ & $x$ & 4 & Tehran, Iran & $\begin{array}{l}2188,2003- \\
2013\end{array}$ & $\begin{array}{l}\text { Cluster } \\
\text { Analysis by } \\
\text { average nearest } \\
\text { neighbour } \\
\text { method and } \\
\text { multiple } \\
\text { regression to } \\
\text { find } \\
\text { associations }\end{array}$ & $\begin{array}{l}7 \\
r \\
r \\
c \\
\epsilon \\
F \\
c \\
\epsilon \\
r \\
r\end{array}$ \\
\hline 36 & $\begin{array}{l}\text { Monti (2016) } \\
(47)\end{array}$ & $\begin{array}{l}\text { Dot density } \\
\text { map }\end{array}$ & $x$ & $\begin{array}{l}\text { Heavy metals, } \\
\text { UV, and } \\
\text { Urbanization }\end{array}$ & $x$ & 2 & $\begin{array}{l}\text { South-Western } \\
\text { Sardinia, Italy }\end{array}$ & $\begin{array}{l}282,2011- \\
2016\end{array}$ & $\begin{array}{l}\text { Multiple logistic } \\
\text { regression were } \\
\text { used to } \\
\text { evaluate } \\
\text { association of } \\
\text { risk factors }\end{array}$ & $\begin{array}{l}s \\
s \\
r \\
k \\
\epsilon \\
\epsilon\end{array}$ \\
\hline 37 & $\begin{array}{l}\text { Lavery (2017) } \\
(48)\end{array}$ & $\begin{array}{l}\text { Proportional } \\
\text { map, } \\
\text { Choropleth } \\
\text { map }\end{array}$ & $x$ & $\begin{array}{l}\text { Environmental } \\
\text { Quality Index } \\
\text { (air, water, } \\
\text { land) }\end{array}$ & $x$ & 2 & USA & $\begin{array}{l}1170,2008- \\
2016\end{array}$ & $\begin{array}{l}\text { Logistic } \\
\text { regression and } \\
\text { Poisson } \\
\text { regression used } \\
\text { to find } \\
\text { associations }\end{array}$ & $\begin{array}{l}f \\
\epsilon \\
f \\
c \\
c \\
t\end{array}$ \\
\hline 38 & $\begin{array}{l}\text { Ashtari (2018) } \\
(49)\end{array}$ & $\begin{array}{l}\text { Dot density } \\
\text { map }\end{array}$ & $x$ & $\begin{array}{l}\text { Air quality } \\
\text { index (AQI) }\end{array}$ & $x$ & 2 & Isfahan, Iran & $\begin{array}{l}2000,2008- \\
2016\end{array}$ & $\begin{array}{l}\text { Unadjusted } \\
\text { logistic } \\
\text { regression to } \\
\text { find } \\
\text { associations } \\
\text { and } \\
\text { interpolation to } \\
\text { estimate } \\
\text { unknown } \\
\text { values of AQI }\end{array}$ & $\begin{array}{l}\mathrm{F} \\
\bar{c} \\
\mathrm{f} \\
\mathrm{E} \\
\mathrm{C} \\
\mathrm{f}\end{array}$ \\
\hline 39 & $\begin{array}{l}\text { Ashtari (2018) } \\
(50)\end{array}$ & $\begin{array}{l}\text { Choropleth } \\
\text { map }\end{array}$ & $x$ & $\begin{array}{l}\text { Height above } \\
\text { sea, average } \\
\text { annual rainfall, } \\
\text { and land use }\end{array}$ & $x$ & 2 & Isfahan, Iran & $\begin{array}{l}2000,2001- \\
2014\end{array}$ & $\begin{array}{l}\text { ANOVA, } \\
\text { independent t- } \\
\text { test and } \\
\text { Kruskal-Wallis } \\
\text { were used to } \\
\text { find } \\
\text { associations }\end{array}$ & $\begin{array}{l}\mathrm{l} \\
\mathrm{i} \\
\bar{c} \\
\hat{c} \\
\bar{c} \\
\bar{c} \\
\mathrm{f}\end{array}$ \\
\hline 40 & $\begin{array}{l}\text { Bains (2010) } \\
(51)\end{array}$ & Heat map & $x$ & $\begin{array}{l}\text { UV radiation } \\
\text { (near-horizon } \\
\text { sunshine) }\end{array}$ & $x$ & 2 & Europe & $\begin{array}{l}\text { 62533, } \\
1950-2010\end{array}$ & $\begin{array}{l}\text { Remote } \\
\text { Sensing for } \\
\text { data gathering } \\
\text { and correlation } \\
\text { analysis to find } \\
\text { association }\end{array}$ & $\begin{array}{l}{[} \\
c \\
c \\
r \\
t\end{array}$ \\
\hline 41 & $\begin{array}{l}\text { Pakdel (2019) } \\
(52)\end{array}$ & $\begin{array}{l}\text { Choropleth } \\
\text { map }\end{array}$ & $x$ & $\begin{array}{l}\text { Human } \\
\text { Development } \\
\text { Index (HDI), } \\
\text { Income and } \\
\text { education. }\end{array}$ & $x$ & 1 & Iran & $\begin{array}{l}\text { Not } \\
\text { mentioned, } \\
\text { 2006-2013 }\end{array}$ & $\begin{array}{l}\text { Linear } \\
\text { regression } \\
\text { analysis to find } \\
\text { associations }\end{array}$ & $\begin{array}{l}s \\
r \\
v \\
k \\
r\end{array}$ \\
\hline 42 & $\begin{array}{l}\text { Culpepper } \\
(2010)(53)\end{array}$ & $\begin{array}{l}\text { Dot density } \\
\text { map, } \\
\text { Proportional } \\
\text { map }\end{array}$ & $x$ & $x$ & & 3 & USA & $\begin{array}{l}19219, \\
2007\end{array}$ & $\begin{array}{l}\text { Measuring } \\
\text { availability and } \\
\text { accessibility of } \\
\text { Veterans Health } \\
\text { Administrations } \\
\text { for MS patients } \\
\text { by travel time } \\
\text { and network } \\
\text { analysis }\end{array}$ & $\begin{array}{l}F \\
t \\
c \\
t \\
t \\
t \\
i\end{array}$ \\
\hline
\end{tabular}




\section{- 3.2.1 Thematic mapping}

Most of the included studies ( $n=40$, or 93\%) employed thematic mapping, in 10 of which it was the only GIS application used (Table1, ID: 1-10). These studies used different types of thematic maps for visualisation, depending on their aim. Of these 40 papers, 31 studies (77\%) used at least one Choropleth map: that is, a thematic map where geographic regions are coloured, shaded or patterned in relation to a value. Five articles (12\%) used heat maps to represent the intensity of an attribute using colours, without using geographical boundaries to group them (Table1, ID: 23, 24, 30, 35, 40). The heat maps were used to visualize continuous variables such as air pollution or UV radiation. Four articles (9\%) contained dot density maps to represent location of MS cases (Table 1, ID: 35, 38), geochemical sampling sites (Table 1, ID: 36) or specialty care providers (Table 1, ID: 42) by point symbols. The least common types of maps were proportional maps, in which a larger symbol represents a higher value (Table 1, ID: 28, 37), and bar graph maps, in which a bar graph represents distribution of a variable in a geographical area (Table 1, ID: 34). Figure 2 is an example of a combined choropleth and proportional map to visualize MS prevalence and number of studies in each country. The type of thematic maps used in each study is available in Table 1.

Most of the papers visualised variables related to disease including MS incidence/ prevalence (Table 1, ID: 3-5, 7-26, 28, 33, 35, 38, 39, 41), mortality/ morbidity rate (Table 1, ID: 1, 2, 17), or hospitalization rate (Table 1, ID: 6). Non-disease related variables were environmental variables (Table 1, ID: 27, 28, 30, $32,34,35,37,39,40$ ) and distribution of services (Table 1, ID: 41, 42).

Four studies employed smoothed incidence rates in their maps (Table 1, ID: 17-20) to improve the accuracy of incidence rates for small areas with few observations, and to remove noise (e.g. random variation) showing the geographical pattern clearer.

\section{- 3.2.2 Spatial cluster detection}

Sixteen papers used spatial cluster detection techniques to identify areas of high-risk (or low-risk) of MS (Table 1, ID: 11-25, 35). Only one study (Table 1, ID: 25 ) employed global clustering methods to assess spatial trends (the tendency of spatial clustering) across the entire study region (55). All of the 16 studies in this group used at least one local clustering method to assess significance of local statistics at each location and to identify the location of spatial clusters (hot spots, cold spots) and spatial outliers (56). Ten of these applied a Bayesian hierarchical modelling technique (Table1, ID: 11-20). Bayesian approach estimates posterior probability (PP) that indicates whether the prevalence (or incidence) rate for each area is significantly lower or greater than a given reference value (57). Also, four studies (Table 1, ID: 19-22) used spatial scan statistics, which is defined as the maximum of likelihood ratio test statistics over a collection of scanning windows (58). A few studies used other spatial local cluster detection techniques like Getis Ord Gi (Table 1, ID: 23, 25) or Anselin Local Morans I (Table 1, ID: 25). In addition, one study employed kernel regression that enables identification of hotspot areas (Table1, ID: 24). One study compared Bayesian modelling method with spatial scan statistics using MS incidence data and suggested that Bayesian modelling can identify geographical variations while controlling for possible confounders (Table 1, ID: 20). The type of spatial clustering techniques used in each study is available in Table 1.

\section{- 3.2.3 Spatial risk factors detection}

The association of environmental and geographical risk factors with spatial distribution of MS was investigated in sixteen studies. These risk factors were modifiable (such as air pollution, water pollution or heavy metals) and non-modifiable (such as ultraviolet (UV) radiation or latitude). UV radiation was the most common risk factor (9 articles) reported by the included studies (Table1, ID: 27-31, 32, 34, 36, 40). These studies used satellite data such as NASA Total Ozone Mapping Spectrometer (TOMS) dataset (59) (Table 1, ID: 27, 29, 30 , 34) or other local and national datasets (Table 1, ID: 28, 32) for measuring UV exposure of geographical areas. Air pollution (Table 1, ID: $26,35,37,38$ ) and heavy metals (Table 1, ID: 33,36$)$ were the next most frequently investigated risk factors. A full list of risk factors is available in Table 1.

Most studies used regression analysis to assess the association between risk factors and disease onset. Four studies used variations of linear regression (Table 1, ID: 28, 30, 35, 41) and three studies applied logistic regression to model a dichotomous dependent variable (Table 1, ID: 36-38). Also, Poisson regression was employed in one study to model count data (Table 1, ID: 37). Other less common methods were correlation analysis (Table1: 40), the KruskalWallis test (Table1, ID: 39) and ANOVA test (Table 1, ID: 39). Interpolation methods like Inverse Distance Weighting (IDW) were employed in some studies (Table 1, ID: 27, 33, 38) for estimating unknown values of environmental exposures based on known values.

\section{- 3.2.4 Health access and planning}

Only two articles focused on health access and planning for MS using GIS tools. One study measured MS patients' access to Veterans Health Administration (VHA) in USA (Table 1, ID: 42). Accessibility of specialty clinics was calculated by proximity analysis using Travel-time buffers.

One study identified the activity and related travel behavior of MS patients (Table 1, ID: 43). This study used Global Positioning System (GPS) tags to monitor MS patients' outdoor activity and travel behavior in relation to disease-related disability. 


\section{- 3.5 Visualisation complexity}

Figure 5 demonstrates the GIS visualisation complexity of the included papers. Thematic mapping with 40 papers was the most scored measure and buffer visualisation with one paper was the lowest (Figure $5 \mathrm{a}$ ). Three studies which received a score of zero, did not have any GIS visualisation. $88 \%$ of studies were scored between one and three and only two studies received a score of four. None of the papers scored five or six (Figure 5b).

\section{Discussion}

This systematic scoping review demonstrates the broad application of GIS and spatial analysis in understanding MS. To the best of our knowledge, this is the first study reviewing evidence on utilization of GIS in MS research. We found that most studies were conducted in Europe, with no study specific to countries in Africa, South America or East-Asia identified. This may be related to the low prevalence of MS in these regions. The study groups defined in this review represent important dimensions of health GIS and spatial epidemiology including thematic mapping, spatial cluster detection, risk factors detection and health access and planning.

Thematic mapping is a basic GIS visualisation method commonly used to visualise the pattern of diseases or health conditions across communities. John Snow's cholera outbreak map is a classic example of GIS visualisation in the health context (60). Choropleth maps were the most common type of thematic map identified in the selected papers in this study. One of the main issues in the use of choropleth maps in visualising aggregated values of point-based attributes is the artificial nature of areal boundaries, known as the "modifiable areal unit problem" (MAUP) (61). In this case, the resulting summary values are influenced by both the shape and scale of the aggregation unit (62). Some of the included studies tried to control this bias by using smaller geographic units or employing smoothing techniques. Interestingly, none of the 31 studies that had used Choropleth maps reported MAUP as a limitation. All of the included papers employed static maps based on retrospective data. Interactive web mapping and real-time dashboards offer policymakers and researchers new visualization capacity to explore the most up-to-date spatial patterns of all MS risk factors, indicators and outcomes at small area level (59). These interactive dashboards would facilitate improved decision making in MS care, and could generate new hypotheses for geographical studies of MS.

Spatial cluster detection plays an important role in identifying local variations and quantifying disease patterns (63). In the selected studies Bayesian modeling was the most frequently used method to identify MS hotspots, possibly because the sample size requirement of Bayesian modeling is generally more relaxed (64). As discussed in six of the included studies, Bayesian modeling is more suitable to uncommon diseases like MS where sample size per area is usually small (Table 1, ID: 11-13, 16-18). Another reason for using Bayesian modelling could be the structure of available data. Most of the included studies used aggregated data of MS cases, making other methods like spatial scan statistics less applicable option for cluster detection (58). Furthermore, Bayesian modeling reduces the impact of MAUP, by decreasing the sensitivity to the size and shape of the boundaries (65-67).

GIS has an important role in quantifying MS risk factors and analysing contextual factors in the MS care ecosystem. The MS care ecosystem refers to all the different levels of the system at which care is provided (micro (patient level), meso (organization level), and macro (state or national level)) as well as relevant contextual variables such as social determinants of health, natural and physical characteristics, and lifestyle factors (68). GIS has a unique capacity to visualise the interaction between these different dimensions of the MS care ecosystem and to highlight care gaps. Data from the different levels of the care system can be collected and imported to GIS systems to link with contextual variables. This enables researchers and MS care providers to bring different sources of data into one system to assist in developing informed decision and policy planning in the MS context. Another advantage of GIS is that it supports the linkage and reattribution of MS disease attributes (i.e., incidence and prevalence) with locational and environmental exposures. This gives researchers and policymakers the capacity to visualise potential links between MS and environmental risk factors. A system thinking approach such as this is recommended to provide a "big picture" of the MS care system and research.

Our findings highlighted that the use of GIS for MS care access and planning was infrequently studied. GIS can quantify and measure accessibility of MS care services, for example, by measuring travel distance from patient locations to the nearest MS care facility $(9,69)$. Use of the new capabilities, such as GPS location, of smart-phones and other wearable devices was under-researched in the MS context. These technologies can improve the quality of life of MS patients by monitoring their activities and providing location-specific guidance.

Few of the included studies contained complex GIS visualisation which could provide a higher level of information (Figure 5). Since there is no formal tool for assessing the complexity of GIS visualisations, we developed an informal assessment tool for this aim. Overlaying GIS layers and using more complex analyses can improve a simple thematic map to make it more informative for policy making.

The applications of GIS techniques in the MS context were very different from those of studies reported in other fields (70-72), particularly in the health access and planning theme. A study by Boyda et al. revealed that of 65 GIS studies in the HIV context in Africa, 21(32\%) were in the category of service implementation (71), whereas our review showed that only 2 of $43(4 \%)$ studies related to health care and planning.

This review has a number of potential limitations. While we conducted a comprehensive search using key databases and hand searching, it is possible that some papers may have been missed. For example, those studies which used thematic mapping but have not included any related search term in the title/or abstract. In addition, we only included papers in English, which means there could be other relevant papers in other languages.

The findings of this review introduce interesting directions for future research. As MS is a chronic disease and MS patients need to receive many healthcare services during their life, more research regarding accessibility of health services and resource management is needed. Furthermore, The novel use of smart phones and GPS tracking technology opens a wide area of using capacity of location data in controlling MS patients

\section{Conclusion}


This review showed that while the number of studies using GIS has increased in the last decade and they mostly employed more than one types of GIS applications, most of these studies used simple types of GIS visualisation. Additionally, the area of health accessibility and planning is under-researched in MS research. Further research is required to support MS researchers to improve their GIS application particularly in the visualisation aspect.

\section{Declarations}

\section{Acknowledgement}

This work was supported by Incubator Grant funded by MS Research Australia (application number: 19-0656). Title of the project: Analysis and mapping of service availability and pattern of care for multiple sclerosis in the Australian Capital Territory region.

Conflict of interest: The authors declare that they have no competing interests.

\section{References}

1. Browne P, Chandraratna D, Angood C, Tremlett H, Baker C, Taylor BV, et al. Atlas of multiple sclerosis 2013: a growing global problem with widespread inequity. Neurology. 2014;83(11):1022-4.

2. Naci H, Fleurence R, Birt J, Duhig A. Economic Burden of Multiple Sclerosis: A Systematic Review of the Literature. PharmacoEconomics. 2010;28(5):36379 .

3. Wallin MT, Culpepper WJ, Nichols E, Bhutta ZA, Gebrehiwot TT, Hay SI, et al. Global, regional, and national burden of multiple sclerosis 1990-2016: a systematic analysis for the Global Burden of Disease Study 2016. Lancet Neurology. 2019;18(3):269-85.

4. Jelinek GA, Marck CH, Weiland TJ, Pereira N, van der Meer DM, Hadgkiss EJ. Latitude, sun exposure and vitamin D supplementation: associations with quality of life and disease outcomes in a large international cohort of people with multiple sclerosis. Bmc Neurology. 2015;15.

5. Reich DS, Lucchinetti CF, Calabresi PA. Multiple Sclerosis. N Engl J Med. 2018;378(2):169-80.

6. Sospedra M, Martin R. IMMUNOLOGY OF MULTIPLE SCLEROSIS. Annual Review of Immunology. 2005;23(1):683-747.

7. Kurland KS, Gorr WL. GIS tutorial for health: ESRI, Inc.; 2007.

8. Cromley EK, McLafferty SL. GIS and public health: Guilford Press; 2011.

9. McLafferty SL. GIS and health care. Annual review of public health. 2003;24(1):25-42.

10. Shabanikiya H, Hashtarkhani S, Bergquist R, Bagheri N, VafaeiNejad R, Amiri-Gholanlou M, et al. Multiple-scale spatial analysis of paediatric, pedestrian road traffic injuries in a major city in North-Eastern Iran 2015-2019. BMC public health. 2020;20:1-11.

11. Mallawaarachchi T, Walker PA, Young MD, Smyth RE, Lynch HS, Dudgeon G. GIS-based integrated modelling systems for natural resource management. Agricultural Systems. 1996;50(2):169-89.

12. Arksey H, O'Malley L. Scoping studies: towards a methodological framework. International journal of social research methodology. 2005;8(1):19-32.

13. Landtblom AM, Riise T, Boiko A, Soderfeldt B. Distribution of multiple sclerosis in Sweden based on mortality and disability compensation statistics. Neuroepidemiology. 2002;21(4):167-79.

14. Llorca J, Guerrero P, Prieto-Salceda D, Dierssen-Sotos T. Mortality of multiple sclerosis in Spain: Demonstration of a north-south gradient. Neuroepidemiology. 2005;24(3):135-40.

15. Yiannakoulias N, Schopflocher DR, Warren SA, Svenson LW. Parkinson's disease, multiple sclerosis and changes of residence in Alberta. Canadian Journal of Neurological Sciences. 2007;34(3):343-8.

16. Bostrom I, Callander M, Kurtzke JF, Landtblom AM. High prevalence of multiple sclerosis in the Swedish county of Varmland. Multiple Sclerosis Journal. 2009;15(11):1253-62.

17. Palmer AJ, Hitchens PL, Simpson S, O'Leary B, Colman S, Taylor BV. A novel method for calculating prevalence of multiple sclerosis in Australia. Multiple Sclerosis Journal. 2013;19(13):1704-11.

18. Ramagopalan SV, Hoang U, Seagroatt V, Handel A, Ebers GC, Giovannoni G, et al. Geography of hospital admissions for multiple sclerosis in England and comparison with the geography of hospital admissions for infectious mononucleosis: a descriptive study. Journal of neurology, neurosurgery, and psychiatry. 2011;82(6):682-7.

19. Risberg G, Aarseth JH, Nyland H, Lauer K, Myhr KM, Midgard R. Prevalence and incidence of multiple sclerosis in Oppland County - a cross-sectional population-based study in a landlocked county of Eastern Norway. Acta Neurologica Scandinavica. 2011;124(4):250-7.

20. Bargagli AM, Colais P, Agabiti N, Mayer F, Buttari F, Centonze D, et al. Prevalence of multiple sclerosis in the Lazio region, Italy: use of an algorithm based on health information systems. Journal of Neurology. 2016;263(4):751-9.

21. McDonald JJ, Weller B, O'Neil M, Waters C, O'Riordan J, Scottish MSRS. Latitudinal variation in MS incidence in Scotland. Multiple Sclerosis and Related Disorders. 2019;32:116-22.

22. Pugliatti M, Solinas G, Sotgiu S, Castiglia P, Rosati G. Multiple sclerosis distribution in northern Sardinia: spatial cluster analysis of prevalence. Neurology. 2002;58(2):277-82.

23. Bergamaschi R, Montomoli C, Candeloro E, Monti MC, Cioccale R, Bernardinelli L, et al. Bayesian mapping of multiple sclerosis prevalence in the province of Pavia, northern Italy. Journal of the Neurological Sciences. 2006;244(1-2):127-31.

24. Cocco E, Sardu C, Massa R, Mamusa E, Musu L, Ferrigno P, et al. Epidemiology of multiple sclerosis in south-western Sardinia. Multiple sclerosis (Houndmills, Basingstoke, England). 2011;17(11):1282-9. 
25. Fromont A, Binquet C, Sauleau EA, Fournel I, Despalins R, Rollot F, et al. National estimate of multiple sclerosis incidence in France (2001-2007). Multiple Sclerosis Journal. 2012;18(8):1108-15.

26. Pivot D, Debouverie M, Grzebyk M, Brassat D, Clanet M, Clavelou P, et al. Geographical Heterogeneity of Multiple Sclerosis Prevalence in France. PloS one. 2016;11(12):e0167556.

27. Bezzini D, Pepe P, Profili F, Meucci G, Ulivelli M, Bartalini S, et al. Multiple sclerosis spatial cluster in Tuscany. Neurological sciences : official journal of the Italian Neurological Society and of the Italian Society of Clinical Neurophysiology. 2017;38(12):2183-7.

28. Bergamaschi R, Monti MC, Trivelli L, Introcaso VP, Mallucci G, Borrelli P, et al. Increased prevalence of multiple sclerosis and clusters of different disease risk in Northern Italy. Neurological Sciences. 2019.

29. Fromont A, Binquet C, Sauleau EA, Fournel I, Bellisario A, Adnet J, et al. Geographic variations of multiple sclerosis in France. Brain : a journal of neurology. 2010;133(Pt 7):1889-99.

30. Green C, Yu BN, Marrie RA. Exploring the implications of small-area variation in the incidence of multiple sclerosis. American journal of epidemiology. 2013;178(7):1059-66.

31. Torabi M, Green C, Yu N, Marrie RA. Application of three focused cluster detection methods to study geographic variation in the incidence of multiple sclerosis in Manitoba, Canada. Neuroepidemiology. 2014;43(1):38-48.

32. Donnan PT, Parratt JD, Wilson SV, Forbes RB, O'Riordan JI, Swingler RJ. Multiple sclerosis in Tayside, Scotland: detection of clusters using a spatial scan statistic. Multiple sclerosis (Houndmills, Basingstoke, England). 2005;11(4):403-8.

33. Turabelidze G, Schootman M, Zhu BP, Malone JL, Horowitz S, Weidinger J, et al. Multiple sclerosis prevalence and possible lead exposure. Journal of the neurological sciences. 2008;269(1-2):158-62.

34. Saei M, Holakouie-Naieni K, Mostafavi E, Sahraian MA, Mahmoodi M, Mansournia MA, et al. Spatial analysis of multiple sclerosis disease in tehran metropolitan zone, Iran, 2001- 2012. Iran J Public Health. 2014;43(5):621-9.

35. Bihrmann K, Nielsen NM, Magyari M, Koch-Henriksen N, Nordsborg RB, Ersboll AK. Small-scale geographical variation in multiple sclerosis: A case-control study using Danish register data 1971-2013. Multiple sclerosis and related disorders. 2018;23:40-5.

36. Wade BJ. Spatial analysis of global prevalence of multiple sclerosis suggests need for an updated prevalence scale. Mult Scler Int. 2014;2014:124578.

37. Gregory AC, Shendell DG, Okosun IS, Gieseker KE. Multiple Sclerosis disease distribution and potential impact of environmental air pollutants in Georgia. Science of the Total Environment. 2008;396(1):42-51.

38. Sloka JS, Pryse-Phillips WEM, Stefanelli M. The relation of ultraviolet radiation and multiple sclerosis in Newfoundland. Canadian Journal of Neurological Sciences. 2008;35(1):69-74.

39. Beretich BD, Beretich TM. Explaining multiple sclerosis prevalence by ultraviolet exposure: a geospatial analysis. Multiple Sclerosis. 2009;15(8):891-8.

40. Amram O, Schuurman N, Randall E, Zhu F, Saeedi J, Rieckmann P, et al. The use of satellite data to measure ultraviolet-B penetrance and its potential association with age of multiple sclerosis onset. Multiple sclerosis and related disorders. 2018;21:30-4.

41. Handel AE, Handunnetthi L, Giovannoni G, Ebers GC, Ramagopalan SV. Genetic and environmental factors and the distribution of multiple sclerosis in Europe. Eur J Neurol. 2010;17(9):1210-4.

42. Taylor BV, Lucas RM, Dear K, Kilpatrick TJ, Pender MP, van der Mei IAF, et al. Latitudinal variation in incidence and type of first central nervous system demyelinating events. Multiple Sclerosis Journal. 2010;16(4):398-405.

43. Ramagopalan SV, Handel AE, Giovannoni G, Siegel SR, Ebers GC, Chaplin G. Relationship of UV exposure to prevalence of multiple sclerosis in England. Neurology. 2011;76(16):1410-4.

44. Tsai CP, Lee CT. Multiple sclerosis incidence associated with the soil lead and arsenic concentrations in Taiwan. PloS one. 2013;8(6):e65911.

45. Schuurman N, Amram O, Saeedi J, Rieckmann P, Yee I, Tremlett H. A proposed methodology to estimate the cumulative life-time UVB exposure using geographic information systems: An application to multiple sclerosis. Multiple sclerosis and related disorders. 2013;2(1):29-35.

46. Heydarpour P, Amini H, Khoshkish S, Seidkhani H, Sahraian MA, Yunesian M. Potential impact of air pollution on multiple sclerosis in Tehran, Iran. Neuroepidemiology. 2014;43(3-4):233-8.

47. Monti MC, Guido D, Montomoli C, Sardu C, Sanna A, Pretti S, et al. Is Geo-Environmental Exposure a Risk Factor for Multiple Sclerosis? A PopulationBased Cross-Sectional Study in South-Western Sardinia. PloS one. 2016;11(9).

48. Lavery AM, Waldman AT, Charles Casper T, Roalstad S, Candee M, Rose J, et al. Examining the contributions of environmental quality to pediatric multiple sclerosis. Multiple sclerosis and related disorders. 2017;18:164-9.

49. Ashtari F, Esmaeil N, Mansourian M, Poursafa P, Mirmosayyeb O, Barzegar M, et al. An 8-year study of people with multiple sclerosis in Isfahan, Iran: Association between environmental air pollutants and severity of disease. Journal of neuroimmunology. 2018;319:106-11.

50. Ashtari F, Karimi A, Delpisheh A, Meamar R, Sayehmiri K, Daliri S. Estimated Incidence Rate of Multiple Sclerosis and Its Relationship with Geographical Factors in Isfahan Province between the Years 2001 and 2014. International journal of preventive medicine. 2018;9:103.

51. Bains W. Exposure of the eyes to near-horizon sunshine may be a trigger for multiple sclerosis. Medical Hypotheses. 2010;74(3):428-32.

52. Pakdel M, Karin Hedström A, Bidkhori M, Hadei M, Kazemi Moghaddam V, Sarmadi M, et al. Do socioeconomic factors affect the prevalence of multiple sclerosis in Iran? Acta Neurologica Scandinavica. 2019;140(5):328-35.

53. Culpepper WJ, 2nd, Cowper-Ripley D, Litt ER, McDowell TY, Hoffman PM. Using geographic information system tools to improve access to MS specialty care in Veterans Health Administration. Journal of rehabilitation research and development. 2010;47(6):583-91. 
54. Neven A, Janssens D, Alders G, Wets G, Van Wijmeersch B, Feys P. Documenting outdoor activity and travel behaviour in persons with neurological conditions using travel diaries and GPS tracking technology: a pilot study in multiple sclerosis. Disability and rehabilitation. 2013;35(20):1718-25.

55. Jacquez GM. Spatial cluster analysis. The handbook of geographic information science. 2008;395(416).

56. Tao R, Thill JC. Spatial cluster detection in spatial flow data. Geographical Analysis. 2016;48(4):355-72.

57. Ellison AM. Bayesian inference in ecology. Ecology letters. 2004;7(6):509-20.

58. Kulldorff M. A spatial scan statistic. Communications in Statistics-Theory and methods. 1997;26(6):1481-96.

59. McPeters RD, Bhartia P, Krueger AJ, Herman JR, Wellemeyer CG, Seftor CJ, et al. Earth probe total ozone mapping spectrometer (TOMS): data products user's guide. 1998.

60. Snow J. On the mode of communication of cholera: John Churchill; 1855.

61. Fotheringham AS, Wong DW. The modifiable areal unit problem in multivariate statistical analysis. Environment and planning A. 1991;23(7):1025-44.

62. Wong DW. The modifiable areal unit problem (MAUP). WorldMinds: geographical perspectives on 100 problems: Springer; 2004. p. 571-5.

63. Mclafferty S. Disease cluster detection methods: Recent developments and public health implications. Annals of GIS. 2015;21(2):127-33.

64. McNeish D. On using Bayesian methods to address small sample problems. Structural Equation Modeling: A Multidisciplinary Journal. 2016;23(5):75073.

65. Bernardinelli L, Clayton D, Montomoli C. Bayesian estimates of disease maps: how important are priors? Statistics in medicine. 1995;14(21-22):2411-31.

66. Xu P, Huang H, Dong N. The modifiable areal unit problem in traffic safety: basic issue, potential solutions and future research. Journal of traffic and transportation engineering (English edition). 2018;5(1):73-82.

67. Lee D, Robertson C, Ramsay C, Pyper K. Quantifying the impact of the modifiable areal unit problem when estimating the health effects of air pollution. Environmetrics. 2020;31(8):e2643.

68. Furst MA, Bagheri N, Salvador-Carulla L. An ecosystems approach to mental health services research. BJPsych International. 2020:1-3.

69. Wang F. Measurement, optimization, and impact of health care accessibility: a methodological review. Annals of the Association of American Geographers. 2012;102(5):1104-12.

70. Nykiforuk $\mathrm{Cl}$, Flaman LM. Geographic information systems (GIS) for health promotion and public health: a review. Health promotion practice. 2011;12(1):63-73.

71. Boyda DC, Holzman SB, Berman A, Grabowski MK, Chang LW. Geographic Information Systems, spatial analysis, and HIV in Africa: A scoping review. PloS one. 2019;14(5):e0216388.

72. Makanga PT, Schuurman N, von Dadelszen P, Firoz T. A scoping review of geographic information systems in maternal health. International Journal of Gynecology \& Obstetrics. 2016;134(1):13-7.

\section{Figures}




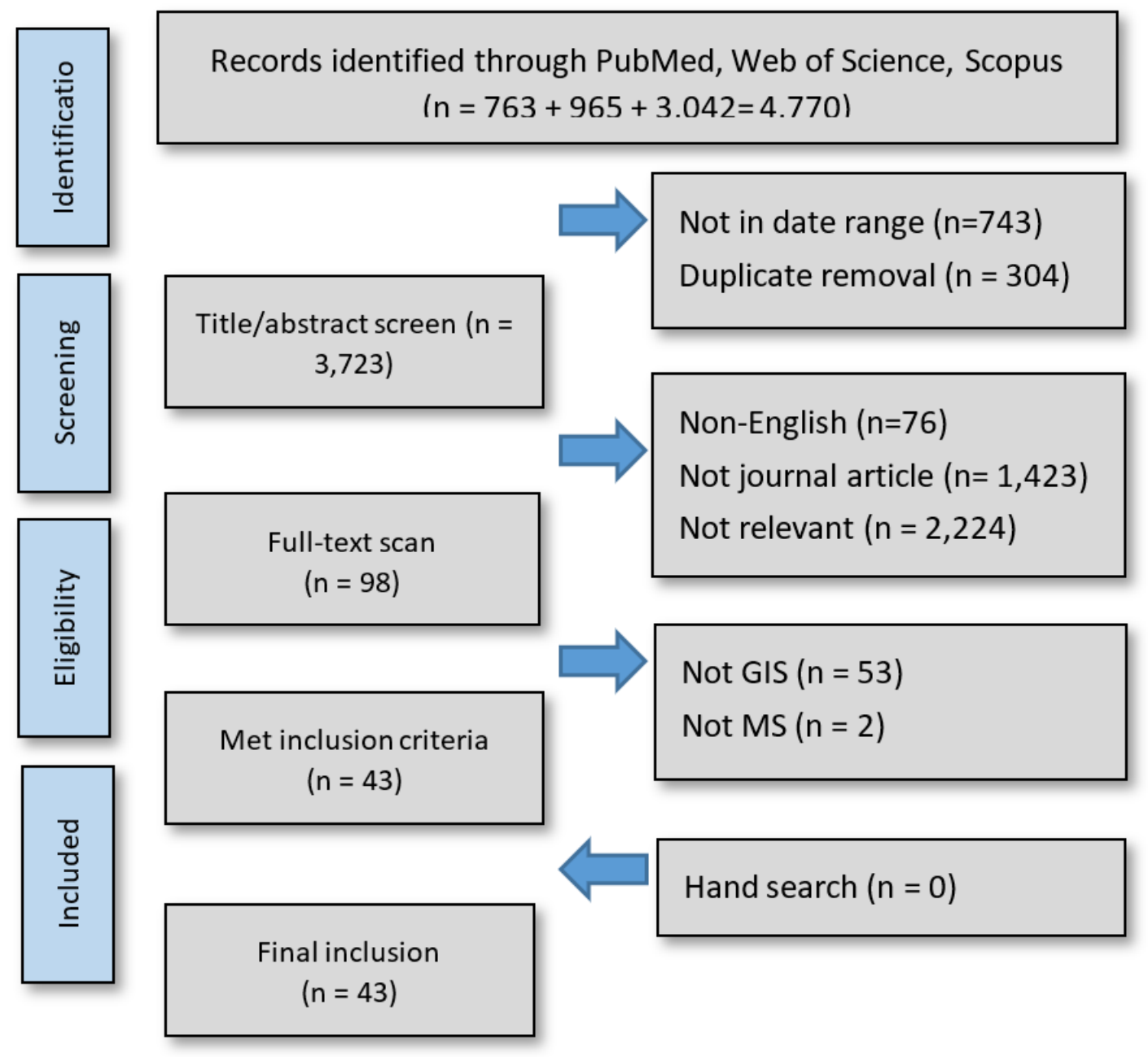

Figure 1

Flowchart of study selection

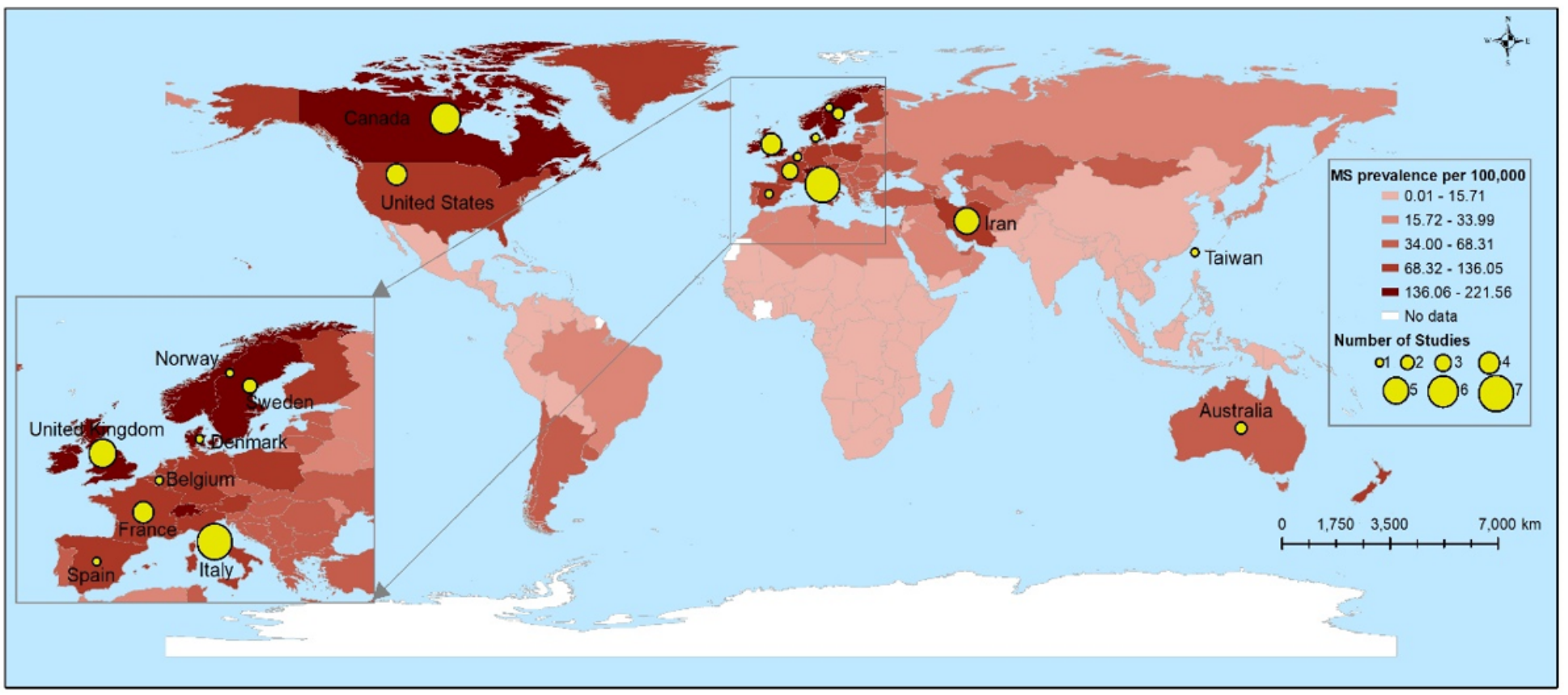

Figure 2 
Geographical variation of single-country studies and prevalence of MS. Note: The designations employed and the presentation of the material on this map do not imply the expression of any opinion whatsoever on the part of Research Square concerning the legal status of any country, territory, city or area or of its authorities, or concerning the delimitation of its frontiers or boundaries. This map has been provided by the authors.

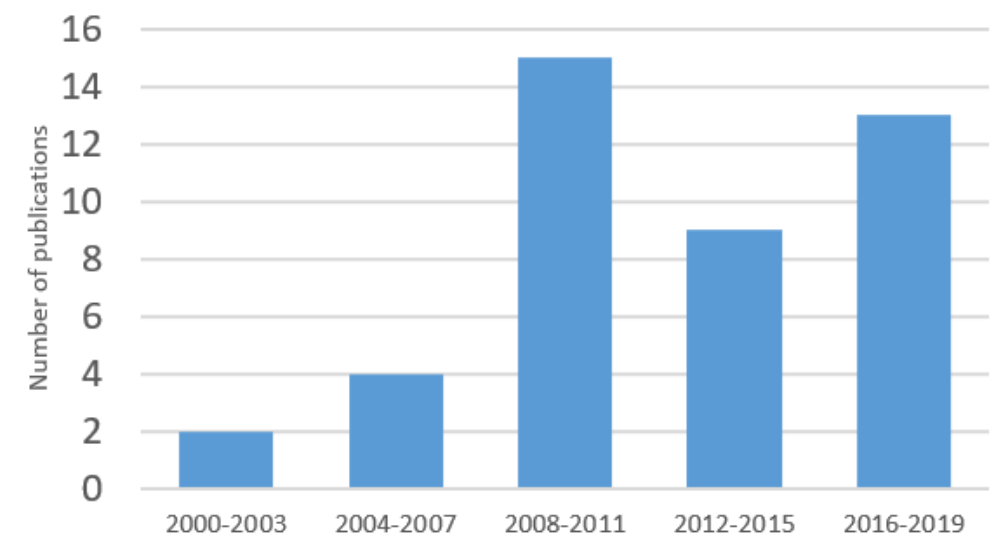

(a)

Time

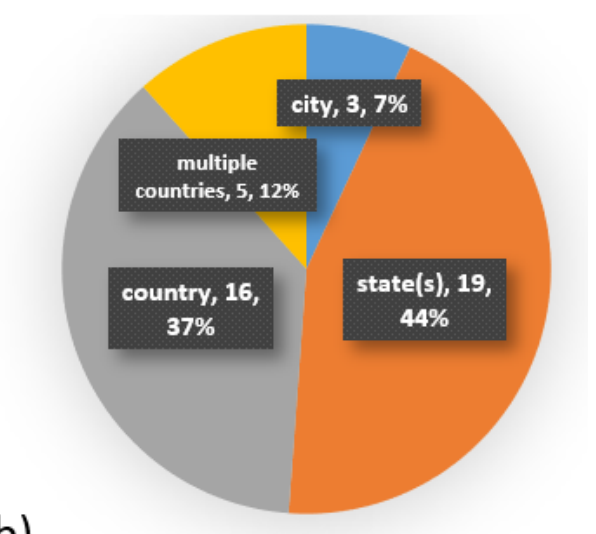

(b)

Figure 3

Distribution of included studies; (a) the trend over time after 2000, (b) geographical scale
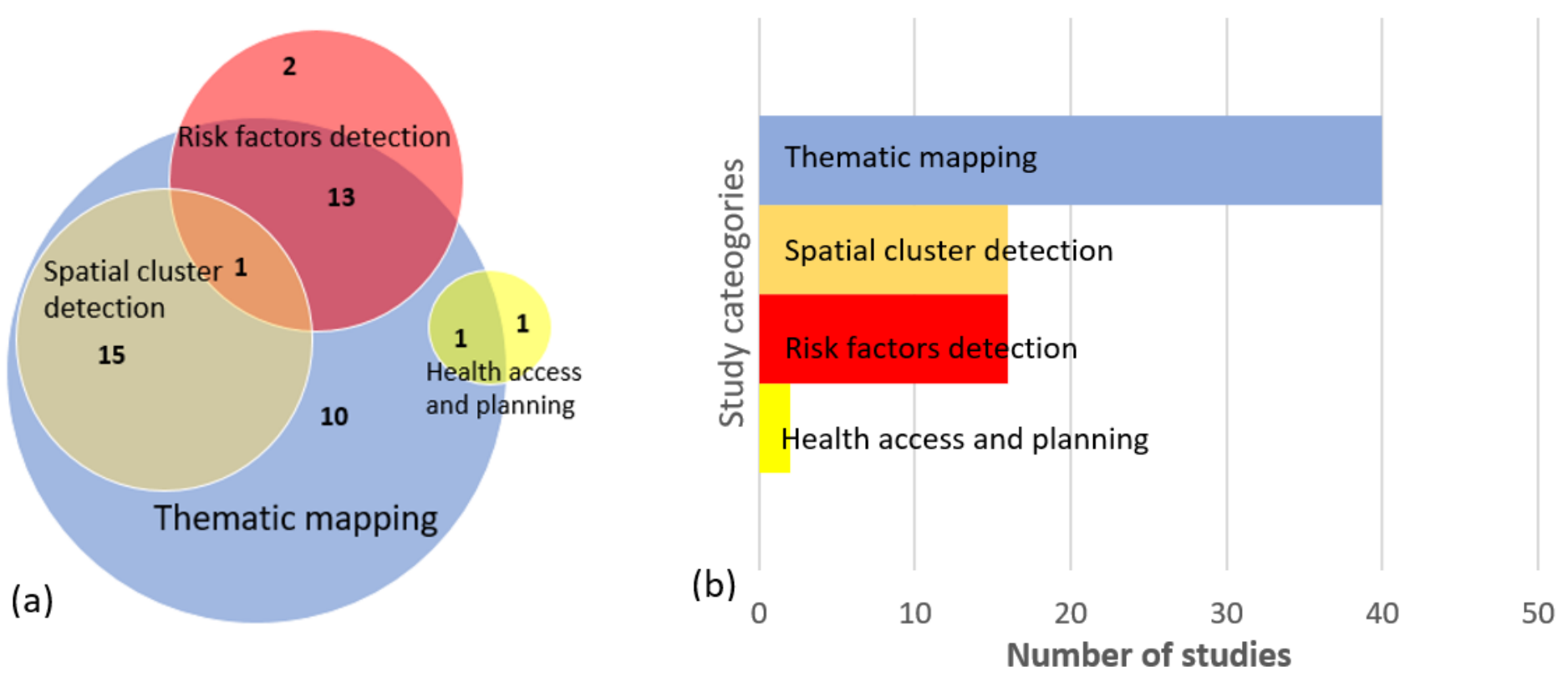

Figure 4

The frequency of the type of used GIS applications; (a) the number and overlap of studies using each type of GIS applications (b) total number of studies in each type of GIS applications 

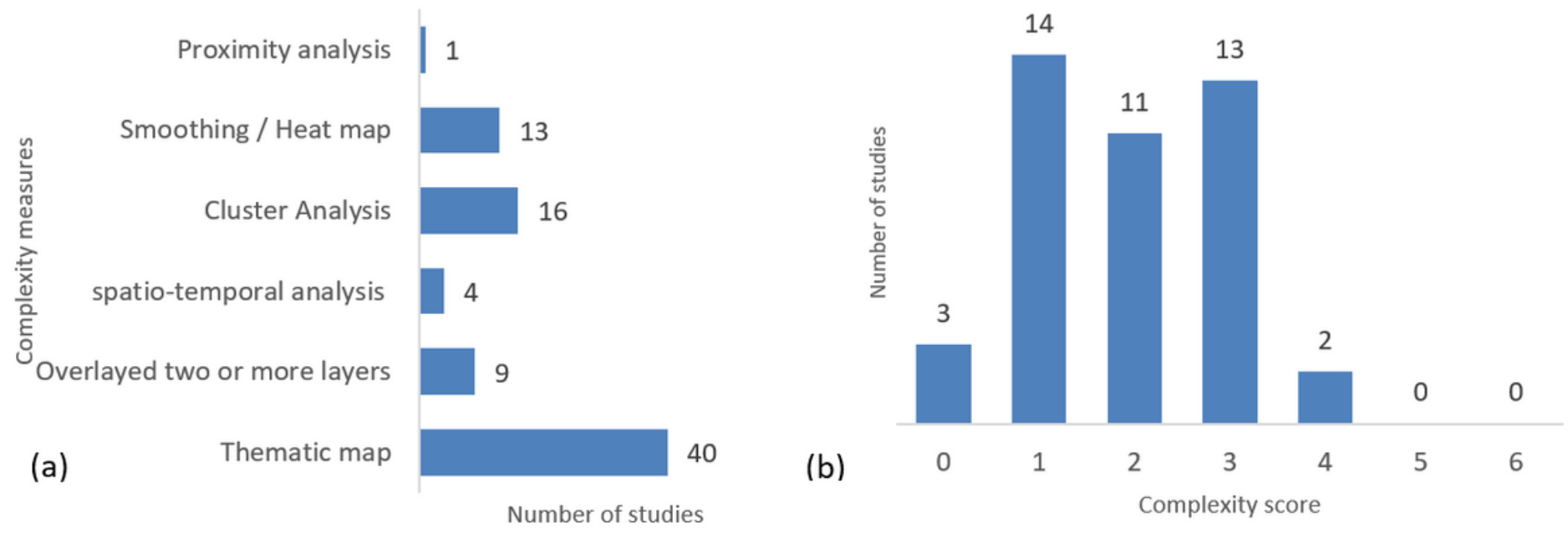

\section{Figure 5}

Complexity scores of included studies; (a) number of studies per measure item (b) total score of included studies 